\title{
Histogram Approaches for Lossy Compression of Digital Holograms of Three-Dimensional Objects
}

\author{
Alison E. Shortt, Thomas J. Naughton, and Bahram Javidi, Fellow, IEEE
}

\begin{abstract}
We present a novel nonuniform quantization compression technique-histogram quantization-for digital holograms of 3-D real-world objects. We exploit a priori knowledge of the distribution of the values in our data. We compare this technique to another histogram based approach: a modified version of Max's algorithm that has been adapted in a straightforward manner to complex-valued 2-D signals. We conclude the compression procedure by applying lossless techniques to our quantized data. We demonstrate improvements over previous results obtained by applying uniform and nonuniform quantization techniques to the hologram data.
\end{abstract}

Index Terms-Digital holography, image compression, nonuniform quantization, 3-D image processing.

\section{INTRODUCTION}

$\mathbf{H}$ OLOGRAPHY is a successful technique for recording and reconstructing 3-D objects. With the advent of digital holography [1]-[7], and continuing advances in megapixel CCD sensors, holograms of useful size and in an appropriate form for processing can be captured. An optical system [8], [9] based on a Mach-Zehnder interferometer [see Fig. 1(a)] was used to record our digital holograms. The resulting digital holograms are in an appropriate form for data transmission and object recognition. It has also been proposed to stream digital holograms over a network to generate a form of 3-D video [10]. The initial stages of such a proposal has involved the compression of individual holographic frames followed by object reconstruction [10]. A real-time 3-D object reconstruction method using the complex field of a digital hologram has been shown to work successfully [11]. A digital hologram comprises several views of the object from a small range of angles. By extracting the appropriate region [12], [13] of pixels from the hologram and by applying a numerical Fresnel propagation technique [2], [5],

Manuscript received April 13, 2006; revised January 31, 2007. This work was supported in part by the Enterprise Ireland and in part by the Science Foundation Ireland. The associate editor coordinating the review of this manuscript and approving it for publication was Prof. Bruno Carpintieri.

A. E. Shortt is with the Department of Computer Science, National University of Ireland, Maynooth, Ireland (e-mail: ashortt@cs.nuim.ie).

T. J. Naughton is with the Department of Computer Science, National University of Ireland, Maynooth, Ireland, and also with the RFMedia Laboratory, University of Oulu, Oulu Southern Institute, 84100 Ylivieska, Finland (e-mail: tomn@cs.nuim.ie).

B. Javidi is with the Electrical and Computer Engineering Department, University of Connecticut, Storrs, CT 06269-2157 USA (e-mail: bahram@engr. uconn.edu).

Color versions of one or more of the figures in this paper are available online at http://ieeexplore.ieee.org.

Digital Object Identifier 10.1109/TIP.2007.894269

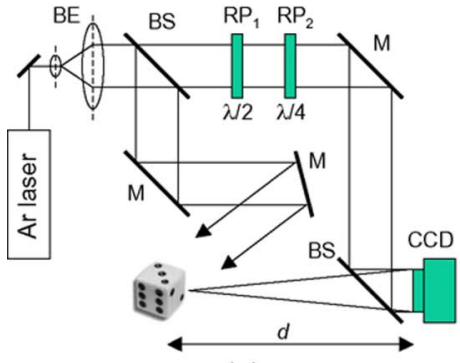

(a)

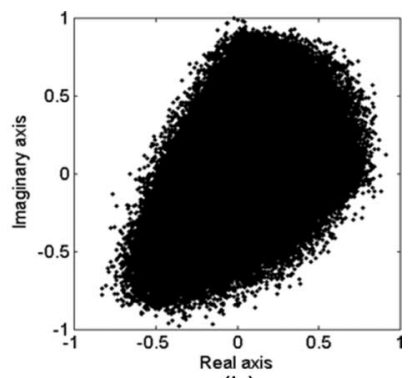

(b)
Fig. 1. (a) Experimental setup for PSI: BE, beam expander; BS, beam splitter; $\mathrm{RP}$, retardation plate; $\mathrm{M}$, mirror, and (b) scatter plot of the complex-valued data in hologram 3 before quantization.

[8], any one of these views of the object can be reconstructed. The dimensions of our digital holograms are $2028 \times 2044$ pixels with each pixel storing 8 bytes of real information and 8 bytes of imaginary information. Given that each hologram is 65 Mbytes in size in its native format, a transmission rate of 2 frames/s over a 1-Gbit/s network connection results. Therefore, realtime holographic video streaming has proven to be impractical over a wide-area network.

Our intention is to compress [14] these holograms for more efficient storage and transmission. Lossless image compression techniques, such as Lempel-Ziv (LZ77,LZW) [15], [16], Huffman [17], and Burrows-Wheeler (BW) [18], perform poorly when applied to holograms due to the inherent speckle content that gives the holograms a white-noise appearance [10]. Holographic speckle is difficult to remove since it actually carries 3-D information. Therefore, the use of lossy compression techniques [10] to initially quantize the holograms, followed by the application of lossless techniques to the quantized holograms, seems essential.

Quantization and phase quantization have been applied successfully to Fourier and holographic data in the past [10], [11], [19]-[27]. In this paper, we quantize our digital holograms using a novel histogram-based technique (that we refer to as histogram quantization) in which we exploit our knowledge of the distribution of the complex values in our data. Uniform quantization was implemented previously since it is an extremely fast and simple technique [10], [20]. Nonuniform quantization achieves superior compression ratios, but it is time consuming due to its iterative nature. Our histogram quantization technique, like uniform quantization, requires just one pass through the data but exhibits the improved results of nonuniform quantization. We extract the cluster centers from histogram plots of the real and imaginary digital hologram data, as well as from histogram plots of the amplitude and phase digital hologram data. We quantize 
our holograms with the resulting cluster centers (a small representative set of complex-valued points used to replace a significantly larger set of input data points).

We compare our histogram quantization approach to another histogram-based technique adapted from what in communications theory is known as optimal signal quantization. This algorithm is known as Max's algorithm [31] and under very specific conditions (scalar quantization, a specific error metric, and realvalued 1-D signals) is optimal. We do not claim that our straightforward adaptation of this technique is optimal for digital holograms, but it acts as a useful benchmark for histogram quantization due to its similar approach and computational complexity. Max's algorithm (or the Lloyd-Max algorithm) has been rediscovered and refined over the years [32]. Max's algorithm was later used for quantization of Fourier spectra for digital hologram applications [33] and it has also been used in the design of an optimally quantized phase-only matched filter for image recognition [34]. We extend Gallagher's innovative work [33] in the following respects: we consider nonuniform step sizes rather than simply finding the optimal uniform step-size quantizer, we consider digital holograms of real-world objects rather than computer-generated objects, and we consider Fresnel holograms rather than Fourier holograms. The problem of quantizing digital holograms of real-world objects is more complicated than that of quantizing (or binarizing) computer-generated holograms in three important respects. First, the often obvious fringe structure in computer-generated holograms is not present in digital holograms of diffuse real-world 3-D objects. This means there is less structure in the hologram that can be exploited by lossless compression techniques. Second, speckle noise is present in real-world holograms. This multiplicative data-dependent noise is very difficult to reduce and can cause many conventional image processing applications to fail. Third, because digital holograms are captured using a physical system as opposed to an idealized system in computer-generated holography, there can be many nonuniformities in the data. One simple example would be that the mean intensity is often spatially dependent in a digital hologram: if one's reference beam does not have perfectly uniform intensity then regions of the digital hologram can be brighter than others. Using the simplest binarization technique as an example, this means that a single intensity-based scalar threshold for a computer-generated hologram would need to be replaced by a complicated nonuniform spatially dependent 2-D threshold function to do the equivalent task with an optically sensed digital hologram.

We use modified Max's algorithm (mMa) quantization to extract the cluster centers from histogram plots of our real and imaginary digital hologram data. We compare the results of our noniterative histogram and mMa quantization techniques with previous uniform quantization [10], [21], [26] and nonuniform quantization ( $k$-means clustering [30], [35], the Kohonen competitive neural network [29], [36], and companding quantization [28]) results. Compression noise or artifacts that emerge in the decompressed hologram are not of immediate concern; rather, of concern is the effect of compression losses on our reconstructed object, range of viewing angles, and so on. We, therefore, use a reconstructed-object-plane rms (root-mean-squared) metric in the reconstruction domain to quantify the quality of our decompressed holograms. We conclude the compression procedure by encoding our quantized digital holograms using lossless compression techniques and measure the amount of compression achieved by each technique for various levels of quantization using compression ratio in the hologram domain.

In Section II, we describe how 3-D objects are captured using digital holography. We discuss the quantization of digital holograms in Section III. We present the results of applying histogram quantization and mMa quantization to our digital holograms in Section IV. In Section V, we complete the compression process by applying lossless techniques to our quantized holographic data and conclude in Section VI.

\section{Phase-Shift Digital Holography}

A technique known as phase-shift interferometry [3], [5] (PSI) was used to create our in-line digital holograms [see Fig. 1(a)] [8], [9]. A linearly polarized Argon ion (514.5 nm) laser beam is expanded and collimated, and divided into object and reference beams. A reference object positioned a distance of approximately $d=350 \mathrm{~mm}$ from a 10-bit $2028 \times 2044$ pixel Kodak Megaplus CCD camera, is illuminated by the first beam. We refer to the complex amplitude distribution in the plane of the object as $U(x, y)$. The linearly polarized reference beam passes through half-wave plate $\mathrm{RP}_{1}$ and quarter-wave plate $\mathrm{RP}_{2}$. This beam can be phase-modulated by rotating the two retardation plates. Through permutation of the fast and slow axes of the plates we can achieve phase shifts of $0,-\pi / 2$, $-\pi$, and $-3 \pi / 2$. The reference beam combines with the light diffracted from the object and forms an interference pattern in the plane of the camera. At each of the four phase shifts we record an interferogram. We use these four real-valued images to compute the camera-plane complex field $H(x, y)$ by PSI [3], [5]. We call this computed field a digital hologram.

A digital hologram $H(x, y)$ contains sufficient amplitude and phase information to reconstruct the complex field $U(x, y, z)$ in a plane in the object beam at any distance $z$ from the camera [5], [8]. This can be calculated using the Fresnel approximation [13], which in convolution form is

$U(x, y, z)=-\frac{\mathrm{i}}{\lambda z} \exp \left(\mathrm{i} \frac{2 \pi}{\lambda} z\right) H(x, y) \star \exp \left[\frac{\mathrm{i} \pi}{\lambda z}\left(x^{2}+y^{2}\right)\right]$

where $\lambda$ is the wavelength of the illumination, and $\star$ denotes a convolution operation. At $z=d$, and ignoring errors in digital propagation due to discrete space (pixelation) and rounding, the digital reconstruction $U(x, y, z)$ approximates $U(x, y)$. Several 3-D objects (see Fig. 2) were used in our experiments. Each had approximate dimensions of $5 \times 5 \times 5 \mathrm{~mm}$ and were positioned $323-420 \mathrm{~mm}$ from the camera.

\section{Digital Hologram Quantization}

Quantization is a data reduction technique that encodes the set of input data values using an appropriate subset of values. Uniform quantization is a data-dependent technique and is the optimal choice when the data values are uniformly distributed. Since our hologram data consists of unevenly distributed complex values, the set of quantization values must be selected carefully in order to account for these biases. When the set of quantization values is a nonuniform sampling of the input data, the 


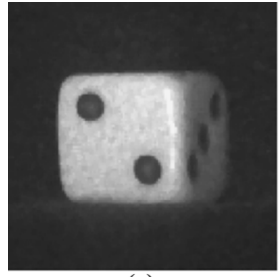

(a)

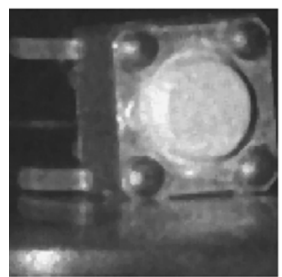

(d))

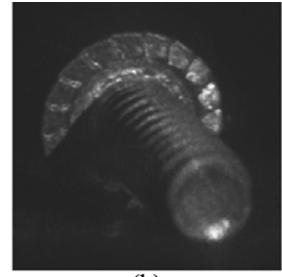

(b)

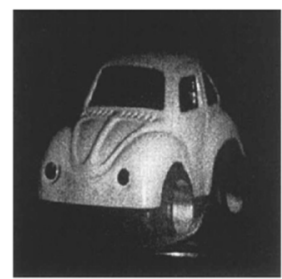

(e)

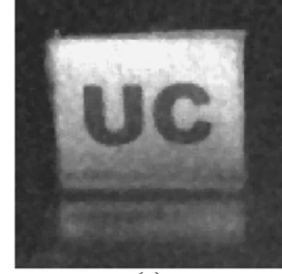

(c)

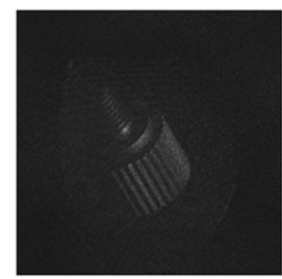

(f)
Fig. 2. Reconstructions from (a) hologram 1, (b) hologram 2, (c) hologram 3, (d) hologram 4, (e) hologram 5, and (f) hologram 6 using unquantized hologram data and with $5 \times 5$ pixel mean filtering.

quantization is referred to as nonuniform quantization. Combined rescaling and uniform quantization using $N$ clusters of a hologram $H$, where $\sqrt{N} \in \mathbb{N}$ and $\sqrt{N}$ is odd, is defined as

$$
H^{\prime}(x, y)=\text { round }\left[H(x, y) \sigma^{-1} \gamma\right] \gamma^{-1}
$$

where

$\sigma=\max \left\{\max _{x, y}|\operatorname{Im}[H(x, y)]|, \max _{x, y}|\operatorname{Re}[H(x, y)]|\right\}$ and $\gamma=(\sqrt{N}-1) / 2$.

Digital holograms have been shown to be particularly sensitive to any defects resulting from lossy compression [10]. Speckle is partly responsible for this; small changes to the digital hologram give rise to the reconstruction of a totally different speckle pattern and so reconstructions differ significantly. Therefore, as a means of reducing the speckle effect, the phase information in the reconstructed object wavefront is discarded and a median or mean filtering operation is applied. Preserving the object plane amplitude information alone is justified since this information has an inherent dependency on both the amplitude and phase of the hologram plane. It follows that if enough amplitude and phase information is preserved in the hologram plane during compression, then the amplitude information in the object plane will be reconstructed properly. The level of acceptable filtering will be application dependent.

In our compression experiments, a digital hologram $H$ is compressed and then decompressed as $H^{\prime}$, and an object $U^{\prime}$ reconstructed by numerical propagation. The quality of the compressed reconstruction $U^{\prime}$ is measured by finding the normalized rms (NRMS) difference between it and the same perspective $U$ from an uncompressed hologram, defined as

$$
\begin{aligned}
& D=\left[\sum_{u=0}^{P_{x}-1} \sum_{v=0}^{P_{y}-1}\left\{|U(u, v)|-\left|U^{\prime}(u, v)\right|\right\}^{2}\right. \\
&\left.\times\left(\sum_{u=0}^{P_{x}-1} \sum_{v=0}^{P_{y}-1}|U(u, v)|^{2}\right)^{-1}\right]^{1 / 2}
\end{aligned}
$$

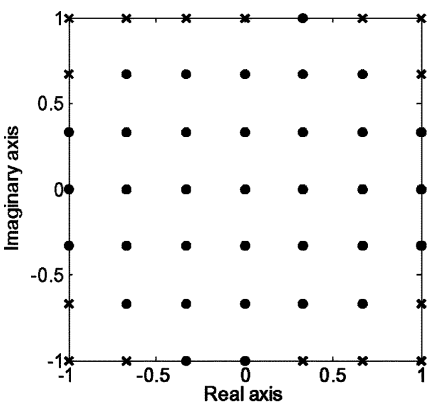

(a)

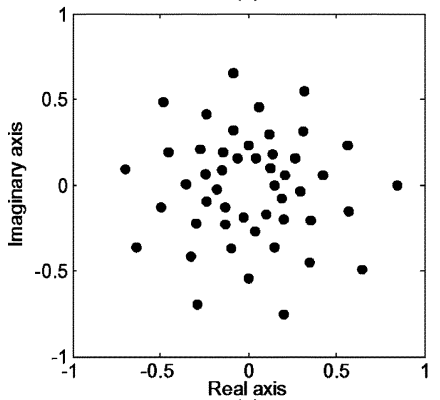

(c)

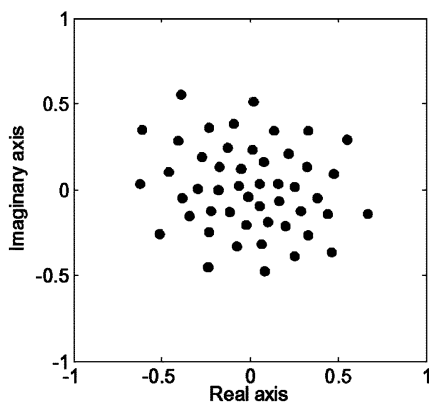

(b)

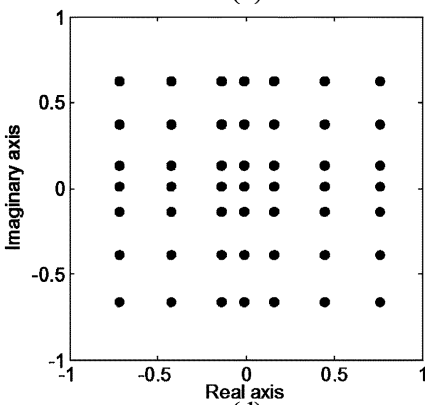

(d)
Fig. 3. Scatter plots of the complex-valued data in hologram 2: (a) uniformly quantized (crosses represent unused clusters), (b) Kohonen competitive, (c) companding quantization, and (d) histogram quantization, all with 49 clusters.

where $P_{x} \times P_{y}$ are the dimensions of the digital hologram and $(u, v)$ are discrete spatial coordinates in the reconstruction plane. In order to reduce the effects of speckle noise, only amplitude in the reconstruction plane is considered and a $5 \times 5$ pixel mean filtering operation is applied prior to calculation of NRMS difference.

\section{NONUNIFORM QUANTIZATION}

The nonuniform distribution of our data, as shown in (1b) for hologram 3, prevents the uniform quantization technique from performing optimally. By nonuniformly positioning the cluster centers to match the fact that there is a higher probability that the pixel will have a low amplitude value, the cluster centers can be used more efficiently.

We previously investigated a number of nonuniform quantization techniques, including $k$-means clustering [30], [35], the Kohonen competitive neural network [29], [36], and companding quantization [28]. Experimental results revealed that these techniques more appropriately positioned cluster centers and produced lower NRMS errors compared to uniform quantization. This is illustrated in Fig. 3(a)-(c), which shows the distribution of clusters relative to the hologram data for uniform quantization, Kohonen competitive, and companding quantization, respectively. Unfortunately, the iterative nonuniform techniques ( $k$-means clustering and Kohonen competitive) are extremely computationally expensive, and, therefore, it is not practical to iterate the algorithms each time a hologram is to be compressed. Ideally, the cluster centers from one class of holograms could be stored in a lookup table and applied with reasonable results to the quantization of subsequent holograms. As might be expected, overfitting occurs when there is too little training data; thus, we previously found that the resulting 


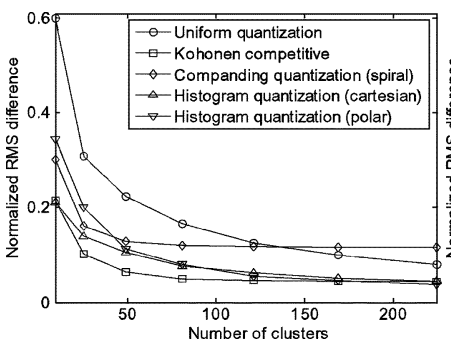

(a)

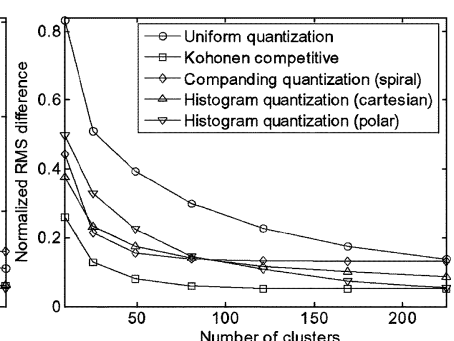

(b)
Fig. 4. NRMS error of the reconstructed object plotted against number of clusters with uniform quantization, Kohonen competitive, companding (spiral) quantization and histogram quantization (in real-imaginary space and amplitude-phase space) for (a) hologram 1 and (b) hologram 2.

cluster centers are significantly less effective when applied in the quantization of a different hologram (see [28, Fig. 1]). Later, we illustrate the effectiveness of our histogram quantization technique when cluster centers from one hologram are used to quantize another hologram.

To speed up the quantization process, we previously developed an approach analogous to companding that involves nonuniformly transforming a fixed-interval sampling grid of the complex plane, rather than transforming the input data. The grid is compressed in regions where the input data is dense (for example, close to the origin) and the grid is stretched in regions where the input data is sparse. The samples are then treated as cluster centers to quantize the input data. We developed two companding grids [28] and found that the spiral, which is based on a logarithmic spiral function, performed best with our digital holograms. Fig. 3(c) shows a spiral companding grid with 49 clusters. In polar coordinates, the spiral has the form $r=a e^{b \theta}$, where $r$ is the distance from the origin, $\theta$ is the angle, and $a$ and $b$ are arbitrary constants. This technique is not only almost as fast as uniform quantization but also consistently produces lower NRMS errors for some numbers of cluster centers, as illustrated in Fig. 4 for two holograms. However, this figure also shows that the iterative technique produces significantly lower NRMS errors compared to both uniform and companding quantization. We now present two quantization techniques that retain the noniterative characteristic of companding quantization, while improving upon its results.

\section{A. Histogram Quantization}

In the noniterative nonuniform quantization technique, we call histogram quantization we can use either histograms of the real and imaginary or amplitude and phase data in the quantization process. Through experimentation, we found that 200 bins in each histogram was sufficient. Initially, we used the $\sqrt{N}$ highest peaks in each of the real and imaginary histograms to define $N$ clusters. However, simply selecting the maxima in the histogram did not prove fruitful. Therefore, for a given complex-valued hologram, we partition the histogram of the real values into $\sqrt{N}$ sections of equal range, where $N$ is the number of clusters (or complex quantization levels) that we desire. This partitioning can be described as follows. Given the set of real or imaginary values in a digital hologram consisting of values in the range $[a, b]$, and a number of sections $N$, the $i$ th section in

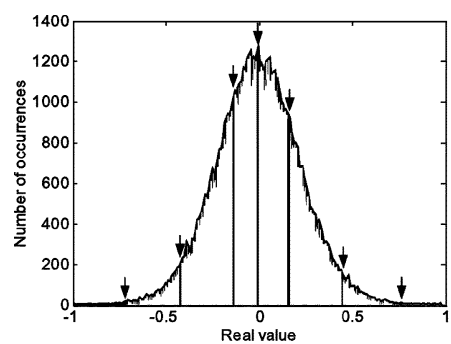

(a)

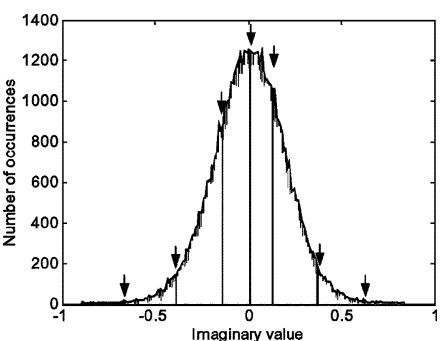

(b)
Fig. 5. Histogram for (a) real and (b) imaginary hologram 2 data with 49 clusters. Arrows denote the seven cluster centers in each dimension.

a partitioning of the histogram of that set would be the range $r_{i}$ defined by

$$
r_{i}= \begin{cases}{[a+i \delta, a+(i+1) \delta),} & \text { if } i<N-1 \\ {[a+i \delta, a+(i+1) \delta],} & \text { if } i=N-1\end{cases}
$$

where $\delta=(b-a) / N$, and where $i \in\{0,1, \ldots, N-1\}$. Next, we extract the value of the highest peak in each range. This highest peak corresponds to the most frequently occurring real or imaginary value in the corresponding range, as shown in Fig. 5. The $\sqrt{N}$ real values and $\sqrt{N}$ imaginary values are paired to give $N$ complex-valued clusters, as shown in Fig. 3(d). Each pixel in the hologram is quantized by changing its value to be the value of its nearest cluster. A higher number of clusters corresponds to increased reconstruction quality and a lower compression ratio. We repeated the procedure with the hologram data in polar (amplitude-phase) representation.

In order to illustrate the effect of codebook size (i.e., the number of clusters) on compression loss, Fig. 4 shows plots of NRMS difference against codebook size for two of the holograms. The Kohonen competitive network consistently outperforms the other techniques over all trials. For smaller codebook sizes, nonuniform quantization is far superior to uniform quantization. As the codebook size increases, this superiority becomes less pronounced. This figure also shows that the histogram quantization technique (using both cartesian and polar space hologram data) achieved better reconstruction results (for the same amount of compression) than those obtained for uniform [10] and companding quantization [28], with histogram quantization performing better in cartesian space than in polar space. With a significant time-complexity advantage (requiring only a single pass) over iterative nonuniform quantization techniques, the noniterative histogram quantization technique (using either cartesian or polar space hologram data) may be used instead of iterative nonuniform techniques in cases where timing is critical.

Fig. 6 illustrates the results of applying the cluster centers resulting from histogram quantization of one hologram to another hologram [referred to as Histogram quantization ("diff. hol.")]. We also include the results of applying histogram quantization directly to the hologram (referred to as Histogram quantization). This figure shows that the cluster centers obtained from the histogram quantization technique (using cartesian space hologram data) are more applicable to unseen hologram data compared to the iterative techniques (see [28, Fig. 1]). This, and comparable results with the other holograms, shows that even when 


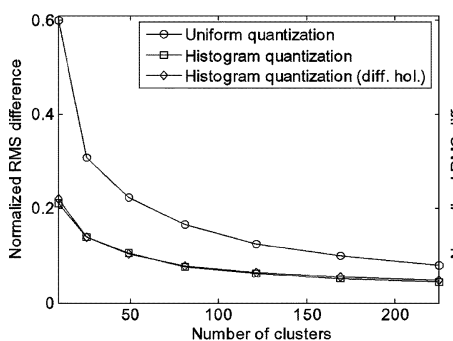

(a)

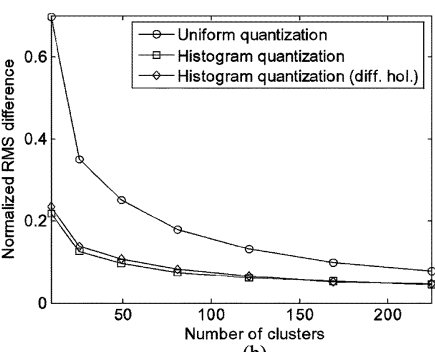

(b)
Fig. 6. NRMS error of the reconstructed object plotted against number of clusters with uniform quantization and histogram quantization for (a) hologram 1. "diff. hol." means using the clusters from hologram 2 to quantize hologram 1 , and (b) hologram 4. "diff. hol." means using the clusters from hologram 3 to quantize hologram 4.

the data set is extremely limited, the over-fitting problem that occurs with the iterative techniques is less likely to occur with the noniterative histogram quantization technique.

\section{B. mMa Quantization}

We now adapt the optimal real-valued 1-D signal quantization technique [31] to complex-valued 2-D signals. In our adaptation of this technique, mMa quantization, which we do not claim is optimal for digital holograms, we apply the approach to histogram plots of the data in cartesian form. Under the specific conditions of Max's study [31], for a quantizer to be considered optimal, it must satisfy the centroid condition and the nearest neighbor condition. The centroid condition

$$
y_{j}=2 \times x_{j}-y_{j-1}, \quad j=2, \ldots, N
$$

where $N$ is the number of quantization levels, $x_{j}$ is a set of end points of the $N$ input ranges, and $y_{j}$ is a set of output or quantization levels corresponding to each input range, states that the optimal quantization level, $y_{j}$ for the $j^{\text {th }}$ region is the center of the inputs that lie in that region. The nearest neighbor condition

$$
\int_{x_{j}}^{x_{j+1}}\left(x-y_{j}\right) p(x) d x=0, \quad j=1, \ldots, N
$$

where $p(x)$ is the input amplitude probability density, states that the $j^{\text {th }}$ region must consist of all inputs closer to codevector $y_{j}$ than any other quantization level [31]. Max [31] attempts to satisfy these conditions and suggests "to pick $y_{1}$, calculate the succeeding $x_{i}$ 's and $y_{i}$ 's and then if $y_{N}$ is the centroid of the area between $x_{N}$ and $\infty, y_{1}$ was chosen correctly." Our pseudocode algorithm mMaOdd (for odd $\mathrm{N}$ ), shown in Fig. 9, is used to quantize one half of the histogram as follows.

1) Set bin equal to $\lfloor$ numBins $/ 2\rfloor+2$ (i.e., index of the next bin to the right of the center bin in $\mathrm{p}$ ).

2) Call mMaOdd.

3) If the area returned from Step 2) is greater than zero then increment bin, then go to Step 2); otherwise, save the $y_{j} \mathrm{~s}$ that are returned from Step 2) and halt. The other half of the histogram is quantized in a similar way.

We implemented this original pseudocode algorithm with Matlab. We used an idealized histogram from Max's paper to test our code: a Gaussian $p(x)=(1 / \sqrt{2 \pi}) e^{-x^{2} / 2}$, with zero mean and standard deviation of 1 . We illustrate the resulting

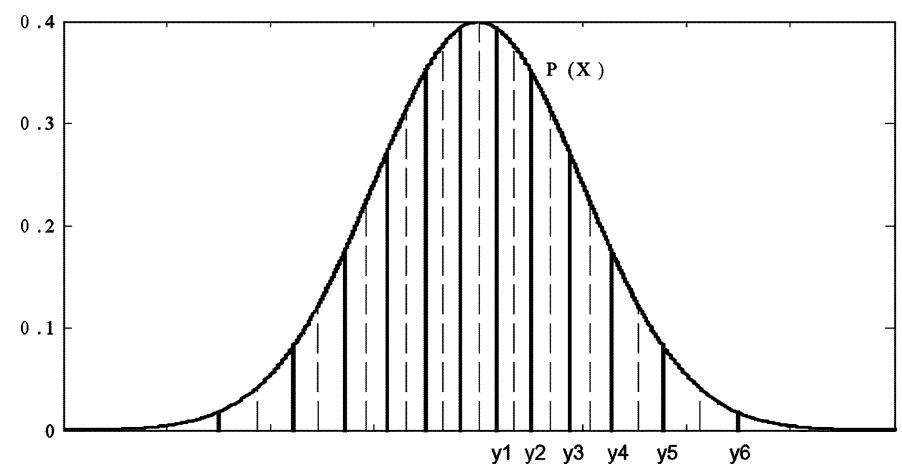

Fig. 7. Gaussian for 12 quantization levels, with solid lines representing quantization levels $\left(y_{j} s\right)$ and dotted lines representing end points of input ranges $\left(x_{j} \mathrm{~s}\right)$.

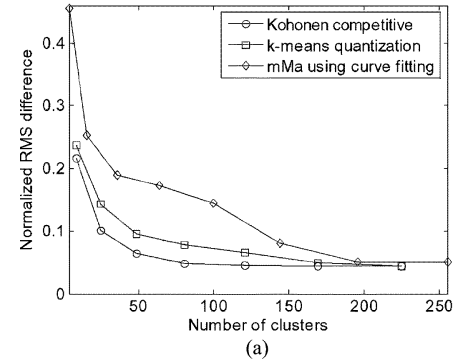

(a)

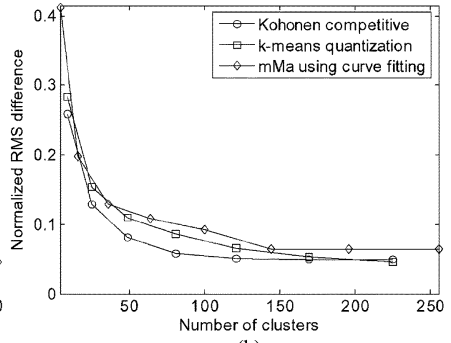

(b)
Fig. 8. NRMS error of the reconstructed object plotted against number of clusters with Kohonen competitive, $k$-means quantization, and mMa using the Gaussian curve fitting approach in (a) hologram 1 and (b) hologram 2.

quantization levels, $y_{j}$, and end points, $x_{j}$, when $N=12$ in Fig. 7, with solid lines corresponding to the $y_{j}$ s and dotted lines corresponding to the $x_{j} \mathrm{~s}$. We performed experiments to determine the appropriate number of bins required to closely match the cluster positions obtained by Max. Using 16001 bins for $p(x)$, our results were within two decimal places of Max's results. Decreasing the number of points to 401 resulted in a 200 -fold decrease in computation time (7420 versus $37 \mathrm{~ms}$, respectively, on our hardware) with results that were still comparable with Max's.

Although a normal distribution is not a precondition for Max's approach, as it happens, our data appeared to be closely approximated by a Gaussian. For a given complex-valued hologram, we calculated the mean and standard deviation of each of the real and imaginary histograms and used these to estimate a Gaussian fit to our data. We used mMa quantization to locate the most appropriate $\sqrt{N}$ quantization levels in each of these real and imaginary Gaussians. We compared our mMa using the Gaussian curve fitting approach to $k$-means quantization and the Kohonen competitive network, as illustrated in Fig. 8. We found that for some holograms, mMa using Gaussian curve fitting gave significantly higher quantization errors.

Our implementation of Max's formalism is capable of working with a realistic discrete histogram that is neither unimodal nor locally smooth. We decided to feed the real and imaginary histograms directly into our algorithm. We experimented with different numbers of bins in our histograms and found that 401 bins was the best tradeoff between computation time and accuracy. We then used mMa quantization to locate a range of even and odd numbers of clusters in our 


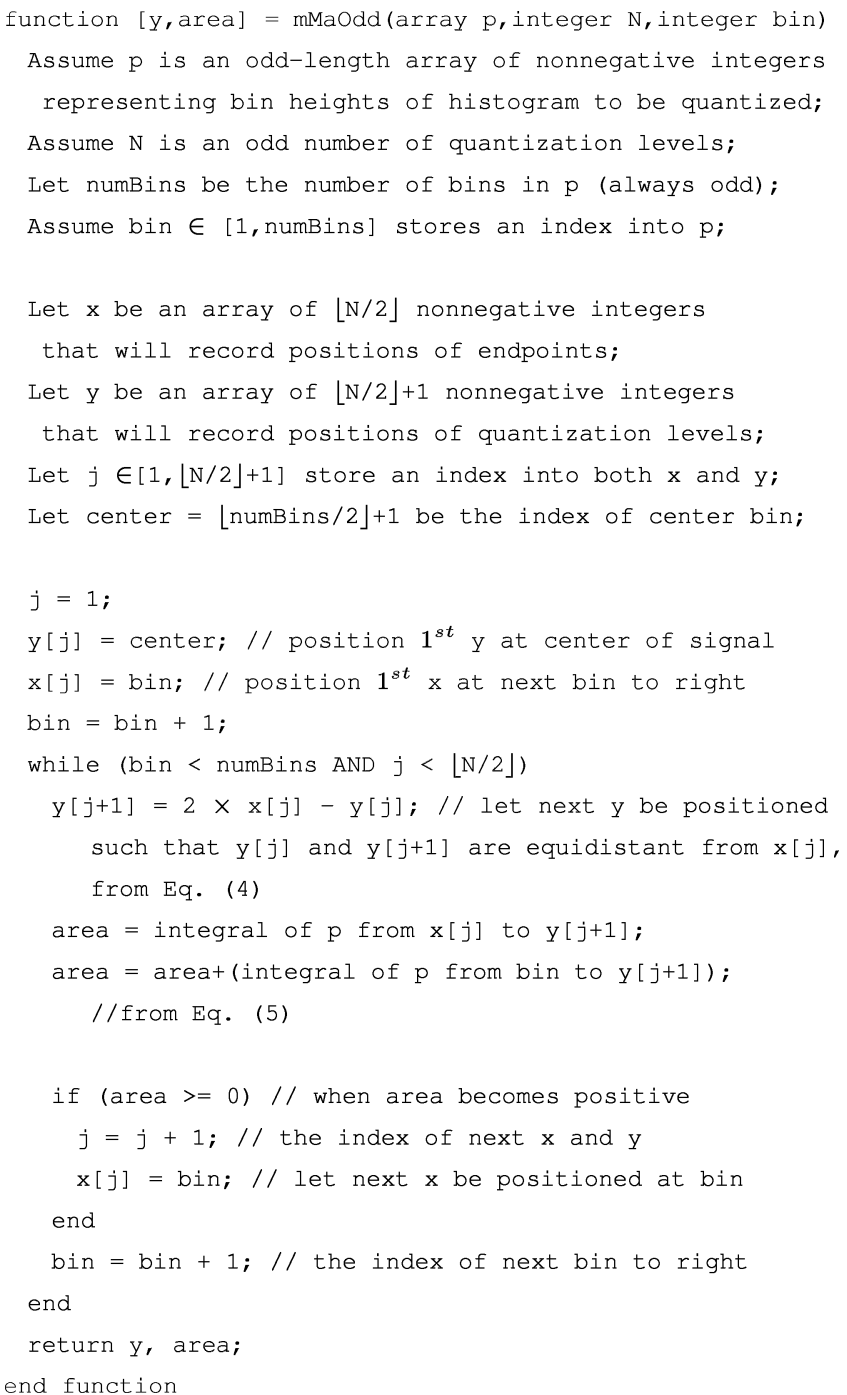

Fig. 9. Pseudocode algorithm for mMa quantization of hologram data based on its histogram. For ease of explanation, the algorithm shown only quantizes one half of the histogram. It can be extended to quantize both sides of the histogram in a straightforward manner.

hologram data. Fig. 10 shows the distribution of 36 clusters relative to the hologram data in two of our holograms. The resulting reconstruction errors for mMa quantization for both even and odd numbers of clusters are shown in Fig. 11 for two holograms. mMa quantization for odd numbers of clusters outperformed both even numbers of clusters and the $k$-means clustering algorithm (ee assume this is due to the histograms' well-defined single mode). However, for numbers of clusters in the $[25,81]$ range, the Kohonen competitive network produced slightly lower NRMS errors compared to mMa quantization consistently for all holograms. There are three reasons for the underperformance of mMa quantization compared to the Kohonen competitive network. mMa quantization assumes slowly varying histograms, but the histograms of our data are not slowly varying. Also, we measure error in the reconstruction domain rather than in the quantized hologram domain. Finally, as with the histogram quantization technique, by separating the real and imaginary components of the complex-valued pixels, the natural correlation between the components is disregarded,

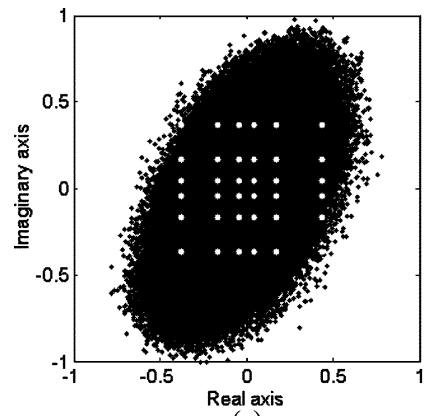

(a)

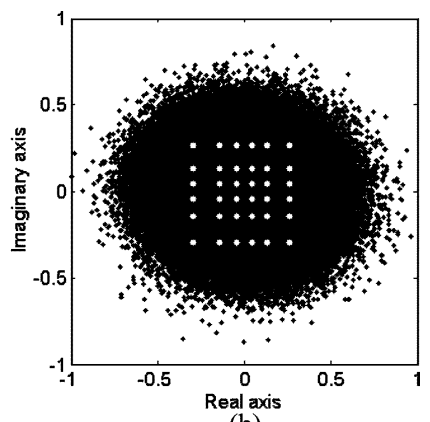

(b)
Fig. 10. Scatter plots of the complex-valued hologram data using 36 clusters resulting from mMa quantization using the histogram approach in (a) hologram 1 and (b) hologram 2.

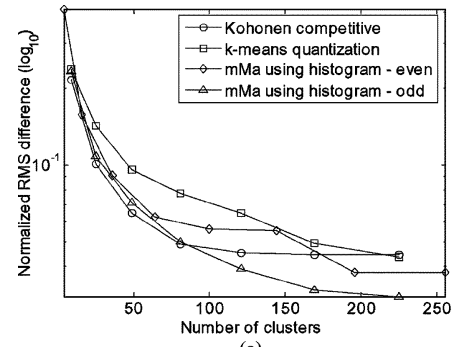

(a)

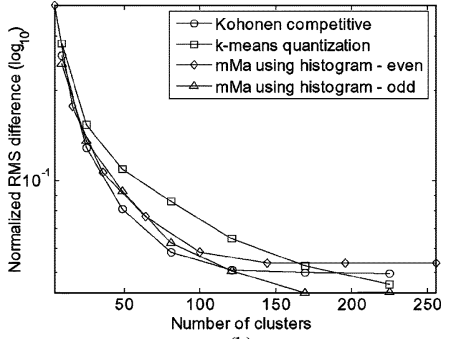

(b)
Fig. 11. NRMS error of the reconstructed object plotted against number of clusters with Kohonen competitive, $k$-means quantization, and mMa quantization using the histogram approach (even and odd) in (a) hologram 1 and (b) hologram 2 .

and this impairs the overall performance of the technique. An advantage of mMa quantization over the Kohonen competitive network is that of speed, since the technique is noniterative.

We compare the performance of the histogram and mMa quantization since both techniques employ a priori knowledge about the distribution of the hologram data. Fig. 12 shows the resulting curves when the reconstruction error was plotted against number of clusters with histogram quantization and mMa quantization applied to a representative selection of the holograms. mMa quantization consistently produced lower NRMS errors (with the exception of nine clusters for some holograms) compared to histogram quantization. However, the running time for histogram quantization is more repeatable than for mMa quantization: for a given number of clusters and data size, histogram quantization takes a constant amount of time, while mMa quantization will take an amount of time that depends on the shape of the histogram and the suitability of the initial starting point.

Reconstructions of two holograms with uniform quantization, the Kohonen competitive network, histogram quantization, and the mMa quantization technique, with each using 9 clusters, are shown in Fig. 13. These can also be compared with the original reconstructions in Fig. 2. There is a significant difference in the quality of the histogram and mMa quantization reconstructions compared to those for uniform quantization. We see little difference in the quality for noniterative quantization compared to iterative quantization.

Table I shows the complexity and running times of each technique to both calculate the cluster centers and quantize the input. $p$ denotes the number of complex-valued pixels in the input, $n$ 


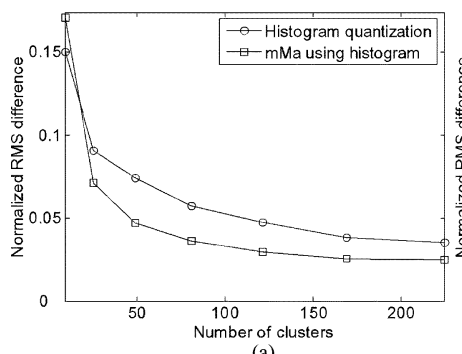

(a)

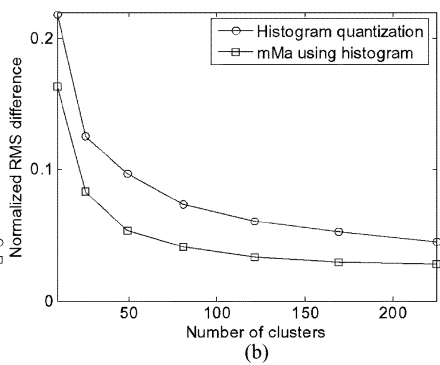

(b)
Fig. 12. NRMS error of the reconstructed object plotted against number of clusters with histogram quantization and mMa quantization using the histogram approach for (a) hologram 3 and (b) hologram 4.

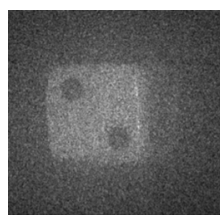

(a)

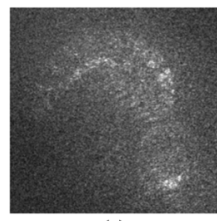

(e)

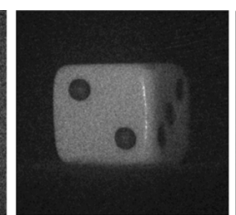

(b)

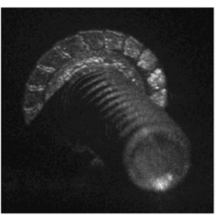

(f)

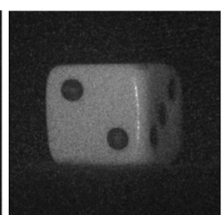

(c)

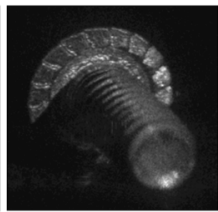

(g)

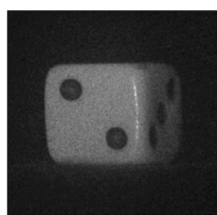

(d)

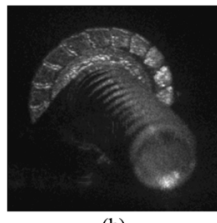

(h)
Fig. 13. Reconstructions with $5 \times 5$ mean filtering in hologram 1 and hologram 2 using (a) and (e) uniform quantization, (b) and (f) Kohonen competitive, (c) and (g) histogram quantization, and (d) and (h) mMa quantization using the histogram approach, all with nine clusters.

TABLE I

COMPLEXITY AND RUNNING TIMES OF THE TECHNIQUES

\begin{tabular}{llll}
\hline Technique & Complexity & Running time (s) & NRMS \\
\hline Uniform & $\Theta(p)$ & 57 & 0.2241 \\
$k$-means & $\Theta(r(p n+p)+p n+n)$ & 168 & 0.0883 \\
Kohonen & $\Theta(r p n+p n)$ & 528 & 0.0615 \\
Companding & $\Theta(p n+n)$ & 438 & 0.1287 \\
mMa & $\Theta(p n+p+n b)$ & 467 & 0.0711 \\
Histogram & $\Theta(p n+p+n+b)$ & 498 & 0.1031 \\
\hline
\end{tabular}

denotes the number of clusters, $r$ denotes the number of iterations, $b$ denotes the number of bins for the histogram techniques, and the asymptotic notation $\Theta(\cdot)$ hides multiplicative and additive constants only. The running times for the noniterative techniques represent direct quantization of the full 2028 $\times 2044$ pixel hologram 1 with 49 clusters. This is too onerous a task for the iterative techniques and so their running times represent training of 49 clusters with a 2048 pixel sample of hologram 1 , and subsequent quantization of the entire hologram. This was the standard procedure used for the iterative techniques throughout the study presented in this paper.

\section{LOSSLESS COMPRESSION OF QUANTIZED Digital HOLOGRAMS}

To complete the compression procedure, standard lossless techniques (Huffman [17], LZ77 [15], LZW [16], and BW [18]) were applied to the quantized digital hologram data. Compression ratio is defined $r=$ (uncompressed size)/(compressed size). In practice, the actual compression ratio employed will be
TABLE II

LOSSLESS COMPRESSION APPLIED TO NONUNIFORMLY QUANTIZED (Histogram) Data From Hologram 4; C.R., COMPRESSION RATIO

\begin{tabular}{llllllllll}
\hline $\begin{array}{l}\text { Clus- } \\
\text { ters }\end{array}$ & $\begin{array}{l}\text { Size } \\
\text { (B) }\end{array}$ & $\begin{array}{l}\text { LZ77 } \\
\text { (B) }\end{array}$ & $\begin{array}{l}\text { LZW } \\
\text { (B) }\end{array}$ & $\begin{array}{l}\text { Huff. } \\
\text { (B) }\end{array}$ & $\begin{array}{l}\text { BW } \\
\text { (B) }\end{array}$ & $\begin{array}{l}\text { LZ77 } \\
\text { c.r. }\end{array}$ & $\begin{array}{l}\text { LZW } \\
\text { c.r. }\end{array}$ & $\begin{array}{l}\text { Huff. } \\
\text { c.r. }\end{array}$ & $\begin{array}{l}\text { BW } \\
\text { c.r. }\end{array}$ \\
\hline 9 & $66,323,721$ & $1,255,190$ & $1,152,210$ & $1,157,893$ & $1,170,881$ & 53 & 58 & 57 & 57 \\
25 & $66,323,721$ & $1,734,374$ & $1,675,201$ & $1,645,957$ & $1,625,298$ & 38 & 40 & 40 & 41 \\
49 & $66,323,721$ & $2,049,159$ & $2,028,363$ & $1,959,436$ & $1,905,904$ & 32 & 33 & 34 & 35 \\
81 & $66,323,721$ & $2,332,206$ & $2,362,175$ & $2,230,057$ & $2,159,921$ & 28 & 28 & 30 & 31 \\
121 & $66,323,721$ & $2,561,802$ & $2,650,336$ & $2,448,604$ & $2,372,901$ & 26 & 25 & 27 & 28 \\
\hline
\end{tabular}

TABLE III

LOSSLESS COMPRESSION APPLIED TO NONUNIFORMLY QUANTIZED (mMa) DATA From HologRAM 4; C.R., COMPRESSION RATIO

\begin{tabular}{llllllllll}
\hline $\begin{array}{l}\text { Clus- } \\
\text { ters }\end{array}$ & $\begin{array}{l}\text { Size } \\
\text { (B) }\end{array}$ & $\begin{array}{l}\text { LZ77 } \\
\text { (B) }\end{array}$ & $\begin{array}{l}\text { LZW } \\
\text { (B) }\end{array}$ & $\begin{array}{l}\text { Huff. } \\
\text { (B) }\end{array}$ & $\begin{array}{l}\text { BW } \\
\text { (B) }\end{array}$ & $\begin{array}{l}\text { LZ77 } \\
\text { c.r. }\end{array}$ & $\begin{array}{l}\text { LZW } \\
\text { c.r. }\end{array}$ & $\begin{array}{l}\text { Huff. } \\
\text { c.r. }\end{array}$ & $\begin{array}{l}\text { BW } \\
\text { c.r. }\end{array}$ \\
\hline 9 & $66,323,721$ & $1,543,454$ & $1,478,327$ & $\mathbf{1 , 4 8 8 , 3 7 6}$ & $\mathbf{1 , 4 5 2 , 3 0 8}$ & 43 & 45 & 45 & 46 \\
25 & $66,323,721$ & $2,125,569$ & $2,111,694$ & $2,031,420$ & $1,971,562$ & 31 & 31 & 33 & 34 \\
49 & $66,323,721$ & $2,555,873$ & $2,640,258$ & $2,444,667$ & $2,365,877$ & 26 & 25 & 27 & 28 \\
81 & $66,323,721$ & $2,945,656$ & $3,232,505$ & $2,878,612$ & $2,783,410$ & 23 & 21 & 23 & 24 \\
121 & $66,323,721$ & $3,165,225$ & $3,622,705$ & $3,139,191$ & $3,047,172$ & 21 & 18 & 21 & 22 \\
\hline
\end{tabular}

dependent on the needs of the user, specifically how much reconstruction loss they are willing to tolerate for their particular application. Tables II and III show the results obtained when hologram 4, quantized using histogram and mMa quantization, respectively, was losslessly compressed using each of the four lossless techniques (hologram 4 gave the lowest compression ratios of all holograms tested). Huffman coding performs least favorably at high compression ratios. For all holograms, and for both histogram and mMa quantization, BW outperforms the other three lossless compression techniques for most numbers of cluster centers. Histogram quantization significantly outperforms mMa quantization in terms of compression ratio for all numbers of cluster centers and for all lossless techniques used in our experiments. The tables also show that as the level of quantization becomes more severe, i.e., as the hologram data is represented by fewer and fewer symbols, the performances of the lossless techniques significantly improve. As was shown in Fig. 13, even for such severe quantization, the reconstructions can be adequate for some applications. Similar results, with compression ratios up to 78 , were obtained for each of the other holograms used in our study.

Looking at the combined compression ratio and NRMS error gives one the full picture. Fig. 14 shows NRMS difference plotted against compression ratio for the $\mathrm{BW}$ technique for histogram quantization, mMa quantization, companding quantization, and the Kohonen competitive network for hologram 1 and hologram 2, respectively. Each point on the curves represents a number of clusters, with the smallest number of clusters represented by the point furthest to the right of each curve. The complete set of such figures (for all holograms) reveals that mMa quantization, with a moderate increase in NRMS error, produces significantly higher compression ratios over each hologram and for each number of clusters compared to the Kohonen competitive network. mMa quantization generally produces comparable compression ratios with companding quantization, but outperforms companding quantization in terms of NRMS error. Histogram quantization produces the 


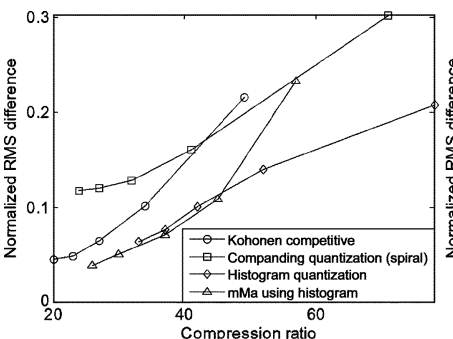

(a)

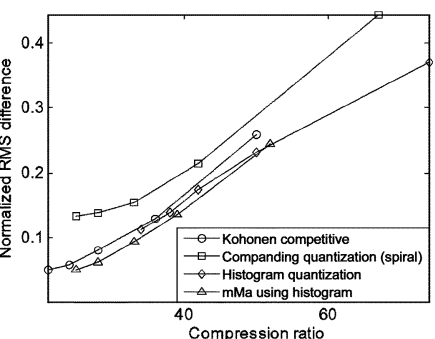

(b)
Fig. 14. NRMS error of the reconstructed object plotted against compression ratio with Kohonen competitive, histogram quantization, and mMa quantization using the histogram approach in (a) hologram 1 and (b) hologram 2, all using the $\mathrm{BW}$ technique.

highest compression ratios for each hologram and for each number of clusters.

\section{CONCLUSION}

We have used two noniterative techniques for the nonuniform quantization of digital holograms of 3-D real-world objects. We compressed the quantized hologram data using lossless techniques. We compared the results of these noniterative techniques to results obtained previously for uniform quantization, iterative nonuniform quantization, and noniterative companding quantization. Performance was evaluated through a NRMS metric in a reconstruction plane and compression ratio in the hologram domain. mMa quantization produced higher compression ratios with slightly higher NRMS errors compared to the iterative techniques. This technique also produced significantly lower NRMS errors compared to companding quantization. The other technique presented in this paper, the histogram quantization technique, outperformed each of the other techniques in terms of compression ratio. We found that $\mathrm{BW}$ compression produced the highest compression ratios among the lossless techniques employed. Compression ratios in the range $[18,78]$ were obtained with good reconstruction quality. In terms of real-time holographic video streaming, the quantization and compression steps could be completed off-line, or if necessary implemented in hardware. With the compression ratios reported here, conventional dedicated communication channels would be sufficient to stream holographic video at 30 frames/s. However, real-time reconstruction of the 3-D objects from the holographic video would require the decompression and Fresnel transform steps to be implemented in dedicated optical [11] or electronic hardware.

\section{ACKNOWLEDGMENT}

The authors would like to thank E. Tajahuerce, Y. Frauel, C. McElhinney, B. Hennelly, and J. McDonald for use of their hologram data.

\section{REFERENCES}

[1] J. W. Goodman and R. W. Lawrence, "Digital image formation from electronically detected holograms," Appl. Phys. Lett., vol. 11, pp. 77-79, 1967.

[2] L. Onural and P. D. Scott, "Digital decoding of in-line holograms," Opt. Eng., vol. 26, no. 11, pp. 1124-1132, 1987.

[3] J. H. Bruning, D. R. Herriott, J. E. Gallagher, D. P. Rosenfeld, A. D. White, and D. J. Brangaccio, "Digital wavefront measuring interferometer for testing optical surfaces and lenses," Appl. Opt., vol. 13, no. 11, pp. 2693-2703, 1974.
[4] U. Schnars and W. P. O. Jüptner, "Direct recording of holograms by a CCD target and numerical reconstruction," Appl. Opt., vol. 33, no. 2, pp. 179-181, 1994.

[5] I. Yamaguchi and T. Zhang, "Phase-shifting digital holography," Opt. Lett., vol. 22, no. 16, pp. 1268-1270, 1997.

[6] E. Cuche, F. Bevilacqua, and C. Depeursinge, "Digital holography for quantitative phase-contrast imaging," Opt. Lett., vol. 24, pp. 291-293, 1999.

[7] T. Kreis, Handbook of Holographic Interferometry: Optical and Digital Methods. Berlin, Germany: Wiley-VCH, 2004

[8] B. Javidi and E. Tajahuerce, "Three-dimensional object recognition by use of digital holography," Opt. Lett., vol. 25, no. 9, pp. 610-612, 2000.

[9] Y. Frauel, E. Tajahuerce, M.-A. Castro, and B. Javidi, "Distortion-tolerant three-dimensional object recognition with digital holography," Appl. Opt., vol. 40, no. 23, pp. 3887-3893, 2001.

[10] T. J. Naughton, Y. Frauel, B. Javidi, and E. Tajahuerce, "Compression of digital holograms for three-dimensional object reconstruction and recognition," Appl. Opt., vol. 41, no. 20, pp. 4124-4132, 2002.

[11] O. Matoba, T. J. Naughton, Y. Frauel, N. Bertaux, and B. Javidi, "Real-time three-dimensional object reconstruction by use of a phase-encoded digital hologram," Appl. Opt., vol. 41, no. 29, pp. 6187-6192, 2002.

[12] H. J. Caulfield, Ed., Handbook of Optical Holography. New York: Academic, 1979.

[13] J. W. Goodman, Introduction to Fourier Optics, 2nd ed. New York: McGraw-Hill, 1996.

[14] M. Rabbani, Ed., Selected Papers on Image Coding and Compression. Bellingham, WA: SPIE, 1992.

[15] J. Ziv and A. Lempel, "A universal algorithm for sequential data compression,” IEEE Trans. Inf. Theory, vol. IT-23, no. 3, pp. 337-343, May 1977.

[16] T. A. Welch, "A technique for high performance data compression," IEEE Computer, vol. 17, no. 6, pp. 8-19, Jun. 1984.

[17] D. A. Huffman, "A method for the construction of minimum redundancy codes," Proc. IRE, vol. 40, pp. 1098-1101, Sep. 1952.

[18] M. Burrows and D. J. Wheeler, "A block-sorting lossless data compression algorithm," Tech. Rep. 124, Digit. Syst. Res. Center, Palo Alto, CA, 1994.

[19] W. J. Dallas and A. W. Lohmann, "Phase quantization in hologramsdepth effects," Appl. Opt., vol. 11, no. 1, pp. 192-194, 1972.

[20] T. Nomura, A. Okazaki, M. Kameda, Y. Morimoto, and B. Javidi, "Image reconstruction from compressed encrypted digital hologram," Opt. Eng., vol. 44, no. 7, p. 075801, 2005.

[21] T. J. Naughton, J. B. Mc. Donald, and B. Javidi, "Efficient compression of Fresnel fields for internet transmission of three-dimensional images," Appl. Opt., vol. 42, no. 23, pp. 4758-4764, 2003.

[22] T. J. Naughton and B. Javidi, "Compression of encrypted three-dimensional objects using digital holography," Opt. Eng., vol. 43, no. 10, pp. 2233-2238, 2004.

[23] D. Kayser, T. Kreis, and W. Jüptner, "Compression of digital holographic data using its electromagnetic field properties," Proc. SPIE, vol. 5908, pp. 97-105, 2005.

[24] I. Yamaguchi, K. Yamamoto, G. A. Mills, and M. Yokota, "Image reconstruction only by phase in phase-shifting digital holography," Appl. Opt., vol. 45, no. 5, pp. 975-983, 2006.

[25] E. Darakis and J. J. Soraghan, "Compression of interference patterns with application to phase-shifting digital holography," Appl. Opt., vol. 45, pp. 2437-2443, 2006.

[26] A. E. Shortt, T. J. Naughton, and B. Javidi, "Compression of digital holograms of three-dimensional objects using wavelets," Opt. Expr., vol. 14, no. 7, pp. 2625-2630, 2006

[27] E. Darakis and J. J. Soraghan, "Use of Fresnelets for phase-shifting digital hologram compression," IEEE Trans. Image Process., vol. 15, no. 12, pp. 3804-3811, Dec. 2006.

[28] A. E. Shortt, T. J. Naughton, and B. Javidi, "A companding approach for nonuniform quantization of digital holograms of three-dimensional objects," Opt. Expr., vol. 14, no. 12, pp. 5129-5134, 2006.

[29] — - "Compression of encrypted digital holograms using artificial neural networks," IEEE/OSA J. Display Technol., vol. 2, no. 4, pp. 401-410, 2006

[30] T. J. Naughton, A. E. Shortt, and B. Javidi, "Nonuniform quantization compression of digital holograms," Opt. Lett., submitted for publication.

[31] J. Max, "Quantizing for minimum distortion," IRE Trans. Inf. Theory, vol. IT-6, pp. 7-12, 1960. 
[32] Y. Linde, A. Buzo, and R. M. Gray, "An algorithm for vector quantizer design," IEEE Trans. Comm., vol. COM-28, no. 1, pp. 84-95, Jan. 1980.

[33] N. C. Gallagher, "Optimum quantization in digital holography," Appl. Opt., vol. 17, no. 1, pp. 109-115, 1978.

[34] V. Kober, L. P. Yaroslavsky, J. Campos, and M. J. Yzuel, "Optimal filter approximation by means of a phase-only filter with quantization," Opt. Lett., vol. 19, no. 13, pp. 978-980, 1994.

[35] J. MacQueen, "Some methods for classification and analysis of multivariate observations," in Proc. 5th Berkeley Symp. Mathematical Statistics and Probability, 1967, vol. 1, pp. 281-297.

[36] T. Kohonen, Self-Organizing Maps. Berlin, Germany: SpringerVerlag, 1994.

Alison E. Shortt received the B.Sc. degree (single honors) in computer science from the National University of Ireland (formerly St. Patrick's College), Maynooth, in 1999, and the Ph.D. degree in 2006.

She is currently a Postdoctoral Researcher at the Department of Electrical and Computer Engineering, University of Connecticut, Storrs. Her research interests include digital holography and compression.
Thomas J. Naughton received the B.Sc. degree (double honors) in computer science and experimental physics from the National University of Ireland (formerly St. Patrick's College), Maynooth, in 1995.

He has worked at Space Technology, Ltd., Ireland, and has been a Visiting Researcher at the Department of Radioelectronics, Czech Technical University, Prague, and the Department of Electrical and Computer Engineering, University of Connecticut, Storrs. He is currently a Lecturer in the Department of Computer Science, National University of Ireland, Maynooth, where he leads research groups in optical information processing, computer theory (optical and biological models of computation), distributed computing, and bioinformatics. He has published over 20 international journal articles and book chapters in these areas.

Mr. Naughton is a member of the Association for Computing Machinery (ACM), the Optical Society of America (OSA), and SPIE.

Bahram Javidi (S'82-M'83-SM'96-F'98) received the B.S. degree in electrical engineering from George Washington University, Washington, DC, and the M.S. and Ph.D. degrees in electrical engineering from the Pennsylvania State University, University Park.

$\mathrm{He}$ is a Board of Trustees Distinguished Professor of Electrical and Computer Engineering with the University of Connecticut, Storrs. He has completed several books, including Optical and Digital Techniques for Information Security (Springer, 2003), Image Recognition: Algorithms, Systems, and Applications (Marcel Dekker, 2002), Three Dimensional Television, Video, and Display Technologies (Springer Verlag, 2002), Smart Imaging Systems (SPIE, 2001), Real-time Optical Information Processing (Academic, 1994), and Optical Pattern Recognition (SPIE, 1994). He has published over 170 technical papers in major journals, including Physics Today and Nature, and his research has been cited in IEEE Spectrum, Science, and New Scientist.

Dr. Javidi is a Fellow OSA and SPIE. He received the IEEE Lasers and Electro-Optics Society Distinguished Lecturer Award in 2003, the IEEE Best Journal Paper Award for the IEEE TRAnsactions on Vehicular TECHNOLOGY in 2002, the University of Connecticut Alumni Association Excellence in Research Award, the Chancellor's Research Excellence Award, and the first Electrical and Computer Engineering Department Outstanding Research Award. 